\title{
Operator Equation and Application of Variation Iterative Method
}

\author{
Ning Chen, Jiqian Chen \\ School of Science, Southwest University of Science and Technology, Mianyang, China \\ Email: chenning783@163.com
}

Received June 8, 2012; revised July 8, 2012; accepted July 15, 2012

\begin{abstract}
In this paper, we study some semi-closed 1-set-contractive operators $A$ and investigate the boundary conditions under which the topological degrees of 1 -set contractive fields, $\operatorname{deg}(I-A, \Omega, p)$ are equal to 1 . Correspondingly, we can obtain some new fixed point theorems for 1-set-contractive operators which extend and improve many famous theorems such as the Leray-Schauder theorem, and operator equation, etc. Lemma 2.1 generalizes the famous theorem. The calculation of topological degrees and index are important things, which combine the existence of solution of for integration and differential equation and or approximation by iteration technique. So, we apply the effective modification of He's variation iteration method to solve some nonlinear and linear equations are proceed to examine some a class of integral-differential equations, to illustrate the effectiveness and convenience of this method.
\end{abstract}

Keywords: Topology Degrees and Index; 1-Set-Contract Operators; Modified Variation Iteration Method; Integral-Differential Equation

\section{Introduction}

In recent years, the fixed point theory and application has rapidly development.

That topological degree theory and fixed point index theory play an important role in the study of fixed points for various classes of nonlinear operators in Banach spaces (see [1-6]). We begin recall theorem A and lemma 1.1 [3]. Then, several new fixed point theorems are obtained in Section 2, and the common solutions of the system of operator equations in Section 3. We also extend some examples for search solution of integral equation and integral-differential equation in Section 4 and Section 5 by variation iterative method. In last part, we compare some figures, by numerical test and note that simple case of Schrodinger equation. The main results are Theorem 2.2, Theorem 3.4-3.5, Example 3, Example 6, etc.

\section{Several Fixed Point Theorems}

Let $E$ be a real Banach space, $\Omega$ a bounded open subset of $E$ and $\theta$ the zero element of $E$.

If $A: \bar{\Omega} \rightarrow E$ is a completely continuous operator, we have some well known theorems as follows (see $[3,4]$ ).

First, we need following some definitions and conclusion (see [3]). For convenience, we first recall theorem A.

Theorem A (see Theorem 1.1 in [3]) Suppose that $A$ has no fixed point on $\partial \Omega$, and one of the following conditions is satisfied,

1) (Leray-Schauder) $\theta \in \Omega, A x \neq \lambda x$, for and $\lambda>1$;

2) (Rothe) $\|A x\| \leq\|x\|$, for all $x \in \partial \Omega$;

3) (Petryshyn) Let $\theta \in \Omega,\|A x\| \leq\|A x-x\|$, for all $x \in \partial \Omega$;

4) (Altman) $\|A x-x\|^{2} \geq\|A x\|^{2}-\|x\|^{2}$, for all $x \in \partial \Omega$. then $\operatorname{deg}(I-A, \Omega, \theta)=1$, and hence $A$ has at least one fixed point in $\bar{\Omega}$.

Lemma 2.1 (see Corollary 2.1 [3]) Let $E$ be a real Banach Space, $\Omega$ is a bounded open subset of $E$ and $\theta \in \Omega$.

If $A: \bar{\Omega} \rightarrow E$ is a semi-closed 1-set-contractive operator such that satisfies the L-S boundary condition

$$
A x \neq t x \text {, for all } x \in \partial \Omega \text { and } t \geq 1,
$$

then $\operatorname{deg}(I-A, \Omega, \theta)=1$, and so $A$ has a fixed point in $\Omega$.

Remark This lemma 2.1 generalizes the famous L-S theorem to the case of semi-closed 1-set-contractive operators.

First, we state following some extend conclusion (see theorem [5]).

Theorem 2.2 Let $E, \Omega, A$ be the same as in lemma 2.1. Moreover, if there exists $\alpha>1, \beta \geq 0, \gamma \geq 0, n$ positive integer such that

$$
\|A x+4 x\|^{n(\alpha+\beta)+\gamma} \leq\|A x+2 x\|^{n \alpha+\gamma}\|A x\|^{n \beta}+\|x\|^{n(\alpha+\beta)+\gamma},
$$


Then $\operatorname{deg}(I-A, \Omega, \theta)=1$ if $A$ has no fixed points on $\partial \Omega$ and so $A$ has a fixed point in $\bar{\Omega}$.

Proof. By lemma 2.1, we can prove theorem 2.2. Suppose that $A$ has no fixed point on $\partial \Omega$.

Then assume it is not true, there exists $x_{0} \in \partial \Omega, \mu_{0} \geq 1$ such that $A x_{0}=\mu_{0} x_{0}$. It is easy to see that $\mu_{0}>1$.

Now, consider the function defined by

$$
f(t)=(t+4)^{n(\alpha+\beta)+\gamma}-(t+2)^{n(\alpha+\beta)+\gamma}-1,
$$

for any $t \geq 1$.

Since

$$
\begin{aligned}
& f^{\prime}(t)=(n \alpha+n \beta+\gamma)(t+4)^{n(\alpha+\beta)+\gamma-1} \\
& -(n \alpha+n \beta+\gamma)(t+2)^{n(\alpha+\beta)+\gamma-1}>0
\end{aligned}
$$

and by formal differential, $f(t)$ is a strictly increasing function in $[1, \infty)$, and so $f(t)>f(1)$ for $t>1$. Thus

$$
(t+4)^{n(\alpha+\beta)+\gamma}>(t+2)^{n(\alpha+\beta)+\gamma}+1>(t+2)^{n \alpha+\gamma} t^{n \beta}+1,
$$

for any $t>1$.

Consequently, noting that $\left\|x_{0}\right\| \neq 0, \quad \mu_{0}>1$, we have

$$
\begin{aligned}
& \left\|A x_{0}+4 x_{0}\right\|^{n(\alpha+\beta)+\gamma}=\left\|\mu_{0} x_{0}+4 x_{0}\right\|^{n(\alpha+\beta)+\gamma} \\
& >\left[(\alpha+2)^{n(\alpha+\beta)+\gamma}+1\right]\left\|x_{0}\right\|^{n(\alpha+\beta)+\gamma} \\
& =\left\|A x_{0}+2 x_{0}\right\|^{n \alpha+\gamma}\left\|A x_{0}\right\|^{n \beta}+\left\|x_{0}\right\|^{n(\alpha+\beta)+\gamma},
\end{aligned}
$$

which contradicts (2.2), and so the condition $(L-S)$ is satisfied. Therefore, it follows from lemma 2.1 that the conclusions of theorem 2.2 hold.

Theorem 2.3 Let $E, \Omega, A$ be the same as in lemma 2.1. Moreover, if there exists $\alpha>1, \beta \geq 0, \gamma \geq 0, m, n$ positive integer such that

$$
\begin{aligned}
& \|A x+(m+1) x\|^{n(\alpha+\beta)+\gamma} \leq\|A x+m x\|^{n \alpha+\gamma}\|A x\|^{n \beta} \\
& +\|x\|^{n(\alpha+\beta)+\gamma}, \text { for all } x \in \partial \Omega .
\end{aligned}
$$

Then $\operatorname{deg}(I-A, \Omega, \theta)=1$, if $A$ has no fixed points on $\partial \Omega$ and so $A$ has a fixed point in $\bar{\Omega}$.

Proof. Similar proof of that theorem 2.2.

Now, we consider the function defined by

$$
f(t)=(t+m+1)^{n(\alpha+\beta)+\gamma}-(t+m)^{n(\alpha+\beta)+\gamma}-1,
$$

for any $t \geq 1$, and $f^{\prime}(t)>0$.

So,

$f(t)$ is a strictly increasing function in $[1,+\infty)$, and $f(t)>f(1)$ for $t>1$. We have

$$
\begin{aligned}
(t+m+1)^{n(\alpha+\beta)+\gamma} & >(t+m)^{n(\alpha+\beta)+\gamma}+1 \\
& >(t+m)^{n \alpha+\gamma} t^{n \beta}+1,
\end{aligned}
$$

for any $t>1$.

Consequently, noting that $\left\|x_{0}\right\| \neq 0, \quad \mu_{0}>1$, we have

$$
\begin{aligned}
\left\|A x_{0}+(m+1) x_{0}\right\|^{n(\alpha+\beta)+\gamma} & >\left\|A x_{0}+m x_{0}\right\|^{n \alpha+\gamma}\left\|A x_{0}\right\|^{n \beta} \\
& +\left\|x_{0}\right\|^{n(\alpha+\beta)+\gamma}
\end{aligned}
$$

which contradicts (2.3). Therefore, it follows from lemma 2.1 that the conclusions of theorem 2.3 hold.

\section{Corollary 2.4 If}

$$
\|A x+(m+1) x\|^{n(\alpha+\beta)+\gamma} \leq\|A x+m x\|^{n \alpha+\gamma}\|A x\|^{n \beta},
$$

then (2.3) holds. By theorem 2.3, A has a fixed point in $\bar{\Omega}$.

We get easy theorem 2.5 in bellow. So, extend (vi) of theorem 2.6 in [3], omit the similar proof.

Theorem 2.5 Let $E, \Omega, A$ be the same as in lemma 2.1. Moreover, if there exists $\alpha \geq 1, \beta \geq 1$ and $m, n$ positive integer such that

$$
\|A x\|^{n \alpha}\|A x+m x\|^{n \beta} \leq\|x\|^{n(\alpha+\beta)} \text {, for all } x \in \partial \Omega .
$$

Then $\operatorname{deg}(I-A, \Omega, \theta)=1$, if $A$ has no fixed points on $\partial \Omega$ and so $A$ has at least one fixed point in $\bar{\Omega}$. (Let $m=1$, that is theorem 2.4 in [5]).

\section{Operator Equations}

We will extend Lemma 2 and Theorem 2, adopt same notation and method in [7] in following form.

Let $E$ be a real Banach space, and $p>1, m$-positive integer.

Lemma 3.1 When $t>1, p>1$ the following holds:

$$
(m+1)(t-1)^{m p}+1 \geq t^{m p}+(1+t)^{m p} .
$$

Proof. Let $f(t)=m t^{m p}-1-(m+1)(t-1)^{m p}+(1+t)^{m p}$, similar the proof of lemma 2 in [7], we easy get $f^{\prime}(t)<0$. In fact, by derivative of it, we have

$$
\begin{aligned}
f^{\prime}(t) & =m(m p) t^{m p-1}-(m+1) m p(t-1)^{m p-1} \\
& +m p(1+t)^{m p-1}, \\
& =m p\left[t^{m p-1}-(m+1)(t-1)^{m p-1}+(1+t)^{m p-1}\right] .
\end{aligned}
$$

Since

$$
\begin{aligned}
& \frac{t^{m p-1}+(1+t)^{m p-1}}{-(m+1)(t-1)^{m p-1}} \\
& =-\frac{1}{(m+1)}\left[\left(\frac{t}{t-1}\right)^{m p-1}+\left(\frac{t+1}{t-1}\right)^{m p-1}\right] \\
& <-\frac{1}{(m+1)}\left(1+\frac{1}{t-1}\right)^{m p-1}-\frac{1}{(m+1)}\left(1+\frac{1}{t-1}\right)^{m p-1} \\
& =-\frac{2}{m+1}<0 .
\end{aligned}
$$

We obtain that 


$$
t^{m p-1}+(1+t)^{m p-1}>(m+1)(t-1)^{m p-1}
$$

that is,

$$
m p\left[t^{m p-1}-(m+1)(t-1)^{m p-1}+(1+t)^{m p-1}\right]>0 .
$$

Thus, $f^{\prime}(t)>0$ Therefore, $f(t)$ is a strictly monotone increasing function in $[0,+\infty)$. When $t>1$ we have $f(t)>f(1)$, and $f(1)=m+2^{m p}>0$, that is $f(t)>0$.

Hence,

$$
t^{m p}+(1+t)^{m p}>(m+1)(t-1)^{m p-1}+1,
$$

where $t>1, p>1$. We complete the proof of this lemma 3.1 .

Theorem 3.2 Let $D$ be a bounded open convex subset in $E$, and $\theta \in D$; Suppose that $A: \bar{D} \rightarrow E$ is a semi-closed 1-set-contradictive operator, and $m, n$-positive integer such that

$$
(m+1)\|A x-x\|^{m p}-\|A x+x\|^{m p} \geq\|A x\|^{m p}-\|x\|^{m p},
$$

for every

$$
x \in \partial D, p>1 .
$$

Then the operator equation $A x=x$ has $a$ solution in $D$.

Proof. By (3.1), we know that $A x=x$ has no solution in $\partial D$, that is $x \neq A x$, for every $x \in \partial D$. We shall prove

$$
x \neq t A x \text {, for every } t \in(0,1) \text {, for every } x \in \partial D \text {. }
$$

In fact, suppose that (3.2) is not true that is there exists a $t_{0} \in(0,1)$, and an $x_{0} \in \partial D$ such that $x_{0}=t_{0} A x_{0}$, that is $A x_{0}=t_{0}^{-1} x_{0}$.

By (3.1), we obtain

$$
\begin{aligned}
& (m+1)\left\|t_{0}^{-1} x_{0}-x_{0}\right\|^{m p}-\left\|t_{0}^{-1} x+x_{0}\right\|^{m p} \\
& \geq\left\|t_{0}^{-1} x_{0}\right\|^{4}-\left\|x_{0}\right\|^{m p},
\end{aligned}
$$

for every $x_{0} \in \partial D, p>1$.

This is because $x_{0} \in \partial D$; hence $\left\|x_{0}\right\| \neq 0$, then we have $(m+1)\left(t_{0}^{-1}-1\right)^{m p}-\left(1+t_{0}^{-1}\right)^{m p} \geq t_{0}^{-m p}-1$.

Let $t=t_{0}^{-1}$, as $t_{0} \in(0,1)$, we have $t>1$.

That is $(m+1)(t-1)^{m p}+1 \geq t^{m p}+(1+t)^{m p}$, then this is a contradiction to Lemma 3.1.

Thus,

$x \neq t A x$, for every $t \in(0,1)$, for every $x \in \partial D$.

From (3.2) and (3.3), we know that $x \neq t A x$. By Ref [6], we obtain that $i(A, D, E)=1$. Then this operator equation $A x=x$ has a solution in $D$.

Theorem 3.4 Let $D$ be a bounded open convex subset in $E$, and $\theta \in D$; Suppose that $A, B: \bar{D} \rightarrow E$ are semi-closed.

1 -set-contradictive operator, and $m, n$-positive integer such that

$$
\begin{aligned}
& (m+1)\|A x-x\|^{m p} \geq\|A x+x\|^{m p}+\|A x\|^{m p} \\
& +\|A x-B x\|^{m p}, \text { for every } x \in \partial D, p>1 .
\end{aligned}
$$

Then the operator equation $A x=x, B x=x$ has $a$ common solution in $D$ (omit the proof of this theorem).

Theorem 3.5 Let Same as assume theorem 3.1. Suppose that $A, B, C: \bar{D} \rightarrow E$ are semi-closed 1-set-contradictive operator, and $m, n$-positive integer, substitute (3.5) for inequality bellow

$$
\begin{aligned}
& (m+1)\|A x-x\|^{m p} \geq\|A x+x\|^{m p}+\|A x\|^{m p} \\
& +\|A x-B x-C x\|^{m p}, x \in \partial D, p>1 .
\end{aligned}
$$

Then the operator equation $A x=x, B x+C x=x$ has a common solution in $D$ (omit this proof).

\section{Solution of Integral Equation}

Recently, the variational iteration method (VIM) has been favorably applied to some various kinds of nonlinear problems, for example, fractional differential equations, nonlinear differential equations, nonlinear thermoelasticity, nonlinear wave equations.

In this section, we apply the variation iteration method (simple writing VIM) to Integral equations bellow (see $[8,9])$. To illustrate the basic idea of the method, we consider:

$$
L[u(t)]+N[u(t)]=g(t) .
$$

The basic character of the method is to construct functional for the system, which reads:

$$
u_{n+1}(x)=u_{n}(x)+\int_{0}^{x} \lambda(s) L u\left[L \bar{u}_{n}+N u_{n}-g(s)\right] \mathrm{d} s
$$

Which can be identified optimally via variation theory, $u_{n}$ is the nth approximate solution, and $\bar{u}_{n}$ denotes a restricted variation, i.e., $\delta \bar{u}_{n}=0$. There is a iterative formula:

$$
u_{n+1}(x)=f(x)+\lambda \int_{a}^{b} k(x, t) u_{n}(t) \mathrm{d} t
$$

of this equation

$$
u(x)=f(x)+\lambda \int_{a}^{b} k(x, t) u(t) \mathrm{d} t
$$

Theorem 4.1 (see theorem 3.1 [8]) Consider the iteration scheme $u_{0}(x)=f(x)$, and

$$
u_{n+1}(x)=f(x)+\lambda \int_{a}^{b} k(x, t) u_{n}(t) \mathrm{d} t
$$

Now, for $n=0,1,2, \cdots$, to construct a sequence of successive iterations that for the $\left\{u_{n}(t)\right\}$ for solution of integral equation $(*)$. 
In addition, we assume that

$$
\int_{a}^{b} \int_{a}^{b} k^{2}(x, t) \mathrm{d} x \mathrm{~d} t=B^{2}<\infty,
$$

and $f(x) \in L_{[a, b]}^{2}$, then if $|\lambda|<1 / B$, the above iteration converges in the norm of $L_{[a, b]}^{2}$ to the solution of integral equation $(*)$.

Corollary 4.2 If $k(x, t)=k_{1}(x, t)+k_{2}(x, t)$, and

$$
\int_{a}^{b} \int_{a}^{b} k^{2}(x, t) \mathrm{d} x \mathrm{~d} t=B^{2}<\infty,
$$

then assume $f(x) \in L_{[a, b]}^{2}$, if $|\lambda|<1 / B$, the above iteration converges in the norm of $L_{[a, b]}^{2}$ to the solution of integral equation $(*)$.

Example 1 Consider that integral equation

$$
u(x)=x^{\alpha}+x^{\beta}+x+\lambda \int_{0}^{1}(x t) u(t) \mathrm{d} t
$$

where $u(0)=0$, and

$$
u_{0}(x)=x^{\alpha}+x^{\beta}+x(0<\alpha<1,0<\beta<1)
$$

From that

$$
u_{n}(x)=x^{\alpha}+x^{\beta}+x+\lambda \int_{0}^{1}(x t) u_{n-1}(t) \mathrm{d} t .
$$

$$
\begin{aligned}
& \text { We have }\left(l=\frac{1}{\alpha+2}+\frac{1}{\beta+2}+\frac{1}{3}\right) \\
& u_{1}(x)=x^{\alpha}+x^{\beta}+x+\lambda \int_{0}^{1}(x t) u_{0}(t) \mathrm{d} t \\
& =x^{\alpha}+x^{\beta}+x+\lambda x l, \\
& u_{2}(x)=x^{\alpha}+x^{\beta}+x+\lambda \int_{0}^{1}(x t) u_{1}(t) \mathrm{d} t \\
& =x^{\alpha}+x^{\beta}+x+\lambda l x\left(1+\frac{\lambda}{3}\right) .
\end{aligned}
$$

From theorem 4.1 and simple computation, we obtain again that

$$
\int_{a}^{b} \int_{a}^{b}(x t)^{2} \mathrm{~d} x \mathrm{~d} t=\int_{0}^{1} \int_{0}^{1}(x t)^{2} \mathrm{~d} x \mathrm{~d} t=\frac{1}{9}=B^{2}<\infty,
$$

and by theorem 4.1 if $|\lambda|<3$, then iterative

$$
u_{n+1}(x)=x^{\alpha}+x^{\beta}+x+\lambda \int_{0}^{1}(x t) u_{n}(t) \mathrm{d} t
$$

is convergent.

Then inductively, we have

$$
\begin{aligned}
u_{n+1}(x) & =x^{\alpha}+x^{\beta}+x+\lambda \int_{0}^{1}(x t) u_{n}(t) \mathrm{d} t \\
& =x^{\alpha}+x^{\beta}+x \\
& +\lambda l x\left[1+\left(\frac{\lambda}{3}\right)+\left(\frac{\lambda}{3}\right)^{2}+\cdots+\left(\frac{\lambda}{3}\right)^{n}\right] .
\end{aligned}
$$

The solution of integral Equation (4.1) by calculating as follows.

$$
\begin{aligned}
& u(x)=\lim _{n \rightarrow \infty} u_{n}(x)=x^{\alpha}+x^{\beta}+x \\
& +\lambda x\left((\alpha+2)^{-1}+(\beta+2)^{-1}+3^{-1}\right)(3 /(3-\lambda)) .
\end{aligned}
$$

Example 2 We consider that integral equation

$$
\begin{aligned}
& u(x)=x^{\alpha}+x^{4}+\lambda \int_{0}^{1}(x t+t) u(t) \mathrm{d} t, \\
& u_{0}(x)=x^{\alpha}+x^{4}(0<\alpha<1) .
\end{aligned}
$$

From $(*)$, we have that

$$
u_{n+1}(x)=x^{\alpha}+x^{4}+\lambda \int_{0}^{1}(x t+t) u_{n}(t) \mathrm{d} t
$$

In fact,

$$
\begin{aligned}
& \int_{a}^{b} \int_{a}^{b} k^{2}(x, t) E x \mathrm{~d} t=\int_{0}^{1} \int_{0}^{1}(x t+t)^{2} \mathrm{~d} x \mathrm{~d} t \\
& =\frac{1}{9}+\frac{1}{3}+\frac{1}{3}=\frac{7}{9}=B^{2}<\infty
\end{aligned}
$$

and by Corollary 4.2 , then if $|\lambda|<3 / \sqrt{7}$, iterative sequence is convergent the solution of Equation (4.2).

\section{Some Effective Modification}

In this section, we apply the effective modification method of He's VIM to solve some integral-differential equations.

In [10] by the variation iteration method (VIM) simulate the system of this form

$$
L u+R u+N u=g(x) .
$$

To illustrate its basic idea of the method .we consider the following general nonlinear system

$$
L u+R u+N u=g(x),
$$

$L u$ the highest derivative and is assumed easily invertible, $R$ is a linear differential operator of order less than $L, N$ represents the nonlinear terms, and $g$ is the source term. Applying the inverse operator $L_{x}^{-1}$ to both sides of Equation (1), and we obtain

$$
u=f-L_{x}^{-1}[R u]-L_{x}^{-1}[N u] .
$$

The variation iteration method (VIM) proposed by Ji-Huan He (see $[5,10]$ has recently been intensively studied by scientists and engineers. the references cited therein) is one of the methods which have received much concern .It is based on the Lagrange multiplier and it merits of simplicity and easy execution. Unlike the traditional numerical methods. Along the direction and technique in [5], we may get more examples bellow.

Example 3 Consider the following integral-differential equation

$$
u^{(5)}(x)=e^{x}-\frac{4}{3} x+\int_{0}^{1} x t u(t) \mathrm{d} t
$$


where $u(0)=1, u^{\prime}(0)=2, u^{\prime \prime}(0)=1, u^{\prime \prime \prime}(0)=1$. In similar example1, we easy have it.

According to the method, we divide $f$ into two parts defined by

$$
\begin{aligned}
& f_{0}(x)=x+e^{x}, u_{0}(x)=f_{0}(x)=x+e^{x}, \\
& f_{1}(x)=-\frac{4}{3(6 !)} x^{6}=-\frac{1}{540} x^{6} .
\end{aligned}
$$

Taking $f(x)=x+e^{x}-(1 / 540) x^{6}$, then we have

$$
\begin{aligned}
u_{1}(x) & =x+e^{x}-(1 / 540) x^{6}+L_{x}^{-1}\left(\int_{0}^{1} x t f_{0}(t) \mathrm{d} t\right) \\
& =x+e^{x},
\end{aligned}
$$

where $L_{x}^{-1}(\cdot)=\int_{0}^{x} \int_{0}^{x} \int_{0}^{x} \int_{0}^{x} \int_{0}^{x}(\cdot) \mathrm{d} t \mathrm{~d} t \mathrm{~d} t \mathrm{~d} t \mathrm{~d} t$, and the processes:

$$
u_{n+1}(x)=x+e^{x}-(1 / 540) x^{6}+L_{x}^{-1}\left(\int_{0}^{1} x t u_{n}(t) \mathrm{d} t\right), n \geq 1 .
$$

Thus, $u_{n+1}(x)=x+e^{x}$, then $u(x)=x+e^{x}$ is the exact solution of (5.1) by only one iteration leads to a solution.

Example 4 (similar example 3 in [5]) Consider the following nonlinear Fredholm integral equation

$$
\begin{aligned}
u(x)= & x-\frac{9 \pi}{12}+\int_{0}^{1} \frac{\sqrt{3}}{3+u^{2}(t)} \mathrm{d} t+\int_{0}^{1} \frac{\sqrt{2}}{2+u^{2}(t)} \\
& +\int_{0}^{1} \frac{1}{1+(u(t) / \sqrt{3})^{2}} \mathrm{~d}\left(\frac{t}{\sqrt{3}}\right) .
\end{aligned}
$$

where from that $\arctan 1=\pi / 4, \quad \arctan \sqrt{3}=\pi / 3$.

$$
u_{0}(x)=f_{0}(x)=x, f_{1}(x)=-(3 \pi / 4), f=f_{0}+f_{1},
$$

by iterative method:

$$
\begin{aligned}
& u_{n+1}(x)=x-\frac{9 \pi}{12}+\int_{0}^{1} \frac{\sqrt{3}}{3+u_{n}^{2}(t)} \mathrm{d} t+\int_{0}^{1} \frac{\sqrt{2}}{2+u_{n}^{2}(t)} \\
& +\int_{0}^{1} \frac{1}{1+\left(u_{n}(t) / \sqrt{3}\right)^{2}} d\left(\frac{t}{\sqrt{3}}\right)=x, n \geq 1 .
\end{aligned}
$$

Clearly, $u(x)=x$ is evident exact solution of (5.2).

\section{Some Notes for Schrodinger Equations}

The quantum mechanics theory and application in more field are widely important meaning.

Along the direction and technique in [11] and [12], we may get more examples.

As we all know the solution of initial problem for Schrodinger equation bellow

$$
\left\{\begin{array}{l}
u_{t}-i a^{2} \Delta u=f(x, t), x \in R^{n}, t>0, \\
u(x, 0)=\varphi(x), x \in R^{n} .
\end{array}\right.
$$

Assume that real part and imaginary part of $\varphi(x), f(x, t)$, are real analytical function for $x \in R^{n}$, then this solution of the problem may express in form:

$$
\begin{aligned}
& u(x, t) \\
& =\sum_{k=0}^{\infty} \frac{\left(i a^{2}\right)^{k}}{k !}\left(t^{k} \Delta^{k} \varphi(x)+\int_{0}^{t}(t-\tau)^{k} \Delta_{x}^{k} f(x, \tau) \mathrm{d} \tau\right) .
\end{aligned}
$$

Now, the authors consider again one-dimension Schrodinger equation as application form:

$$
\begin{aligned}
& \varphi_{x x}(x)+f(x) \varphi(x)=0, x \in R, \\
& f(x)=\frac{2 m}{\tilde{h}}(E-V(x)) .
\end{aligned}
$$

where look in (6.3), that $\varphi(x)$ be the part in space for wave function $\varphi(x, t)$, the $V(x)$ in (6.4) be the potential function $\tilde{h}$ be arrange plank constant, $m$ be the practical mass, $E$ express energy.

The Equation (6.3) for with extensive equation, by calculating and search the general solution that

$$
\varphi(x)=C_{1} \sin (x \sqrt{f(x)})+C_{2} \cos (x \sqrt{f(x)})
$$

So, by (6.3) and with power of (6.4), we consider that two case:

1) (see $[13,14])$ The infinite deep power trap

$$
V(x)=\left(x^{2} / 2\right), E_{n}=\left(\tilde{h}^{2} \pi^{2} / 2 m \alpha^{2}\right) n^{2}, n=0,1,2, \cdots .
$$

2) The shake Power

$$
V(x)=\left(x^{2} / 2\right), E_{n}=(n+0.5) \tilde{h} \omega, n=0,1,2, \cdots .
$$

We take parameters $m=\tilde{h}=\alpha=1, C_{1}=C_{2}=1$.

Then

$$
\begin{aligned}
f(x) & =\frac{2 m}{\tilde{h}^{2}}\left(\frac{\tilde{h}^{2} \pi^{2}}{2 m \alpha^{2}} n^{2}-\frac{x^{2}}{2}\right)=\frac{\pi^{2} n^{2}}{1}-\frac{x^{2}}{2} \\
& =\left(\pi^{2} n^{2}-\frac{x^{2}}{2}\right), n=0,1,2, \cdots .
\end{aligned}
$$

Furthermore, from (6.5), we obtain analytic solution for $\varphi(x)$ and $\phi(x)$. So, we have that

$$
\begin{aligned}
\varphi(x)= & \sin \left(x \sqrt{(\pi n)^{2}-0.5 x^{2}}\right) \\
& +\cos \left(x \sqrt{(\pi n)^{2}-0.5 x^{2}}\right), n=0,1, \cdots \\
\phi(x)= & \sin \left(x \sqrt{2 n+1-x^{2}}\right) \\
& +\cos \left(x \sqrt{2 n+1-x^{2}}\right), n=0,1,2, \cdots .
\end{aligned}
$$

See Figures 1 and $\mathbf{2}$ below.

Therefore, by using of mathematical software with Matlab (see [14]), we may proceed numerical imitate, to get approximate solution, see Figures 3 and 4 . 


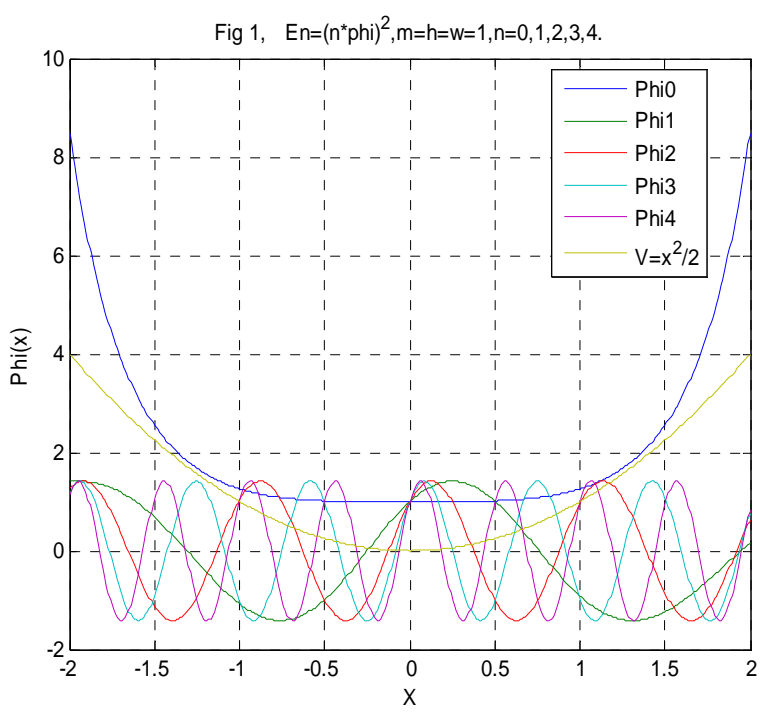

Figure 1. The $\varphi(x)$ is the space form of wave function $\varphi(x . t)$ for (6.3) under action of shake $V(x)=0.5 x^{2}$, by $\varphi_{0}, \varphi_{1}, \cdots, \varphi_{4}$ express for 0-level, 1-level, $\cdots, 4$-level wave function respectively.

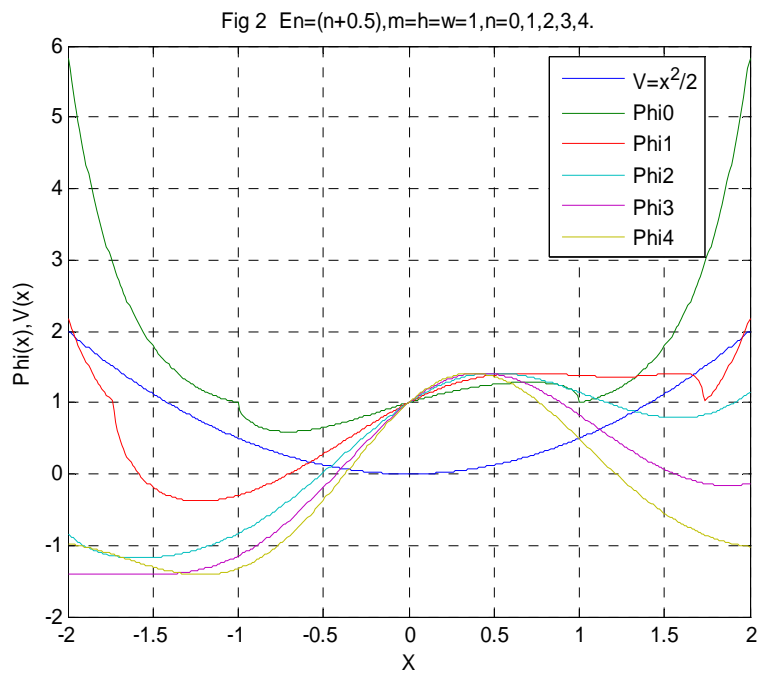

Figure 2. The $\phi(x)$ is the space form of wave function $\phi(x, t)$ for (6.3) under action of shake power $V(x)=0.5 x^{2}$, by $\varphi_{0}$, $\varphi_{1}, \cdots, \varphi_{4}$ express for 0 -level, 1 -level, $\cdots, 4$-level wave function respectively.

In fact, according to the finite difference principle, a one-dimensional Schrodinger equation can be converted into a set of nodal liner equations expressed in a matrix equation after the space is divided into a series of discrete nodes with an equal interval. The matrix left division command offered in the MATLAB software can be used to derive the function approximation of each unknown nodal function.

\section{Concluding Remarks}

In this Letter, we consider operator equations and apply

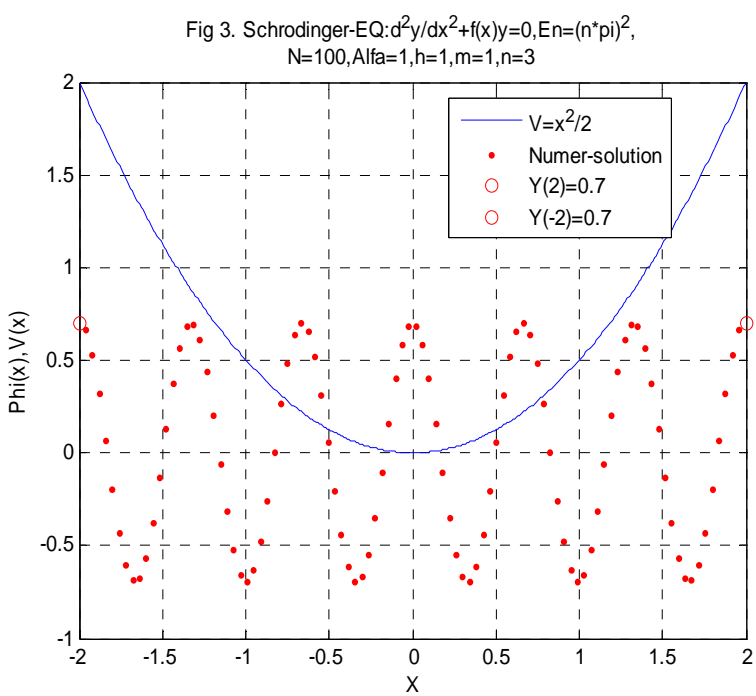

Figure 3. The $\varphi(x)$ is numerical solution by action of (6.3) under the shake power $V(x)=0.5 x^{2}$ and in boundary value condition $\varphi(-2)=\varphi(2)=0.7$, the $\varphi_{3}(x)$ express 3-level (here step length $=0.04$, the energy $E_{n}=\left((n \pi)^{2}, n=3\right)$.

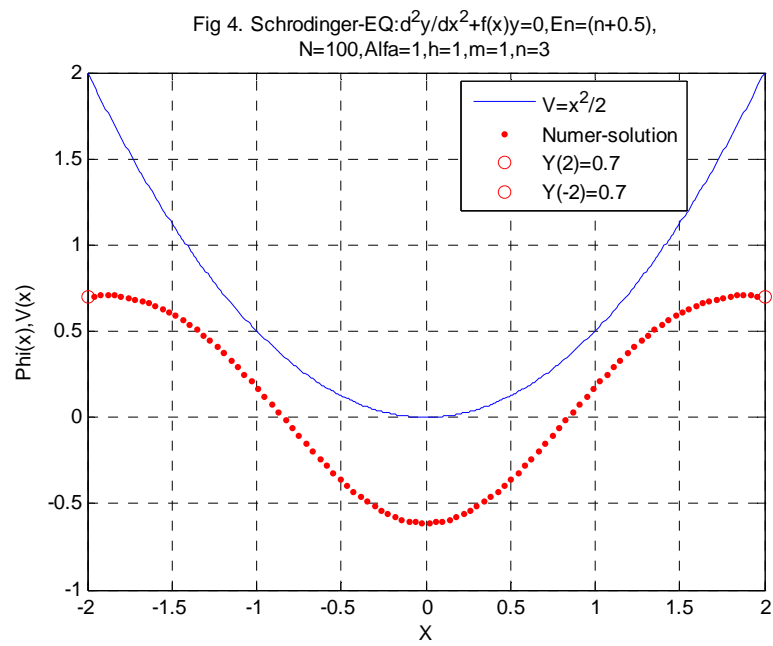

Figure 4. The $\varphi(x)$ is numerical solution by action of (6.3) under the shake power $V(x)=0.5 x^{2}$ and in boundary value condition $\varphi(-2)=\varphi(2)=0.7$, the $\varphi_{3}(x)$ express 3-level (here step length $=0.04$, the energy $E_{n}=\left((n \pi)^{2}, n=3\right)$.

the variation iteration method to integral-differential equations, and extend some results in $[3,8,10]$. The obtained solution shows the method is also a very convenient and effective for various integral-differential equations, only one iteration leads to exact solutions. Recently, the impulsive differential delay equations is also a very interesting topic, and we may see [10] etc.

In our future work, we may try to do some research in this field and may be could obtain some better results.

\section{Acknowledgements}

This work is supported by the Natural Science Founda- 
tion (No. 11ZB192) of Sichuan Education Bureau and the key program of Science and Technology Foundation (No. 11ZD1007) of Southwest University of Science and Technology.

The author thanks the Editor kindest suggestions, and thanks the referee for his comments.

\section{REFERENCES}

[1] D. Guo and V. Lashmikantham, "Nonlinear Problems in abstract Cones," Academic Press, Inc., Boston, New York, 1988.

[2] Y. J. Cui, F. Wang and Y. M. Zou, "Computation for the Fixed Index and Its Applications," Nonlinear Analysis, Vol. 71, No. 1-2, 2009, pp. 219-226. doi:10.1016/j.na.2008.10.041

[3] S. Y. Xu, "New Fixed Point Theorems for 1-Set-Contractive Operators in Banach Spaces," Nonlinear Analysis, Vol. 67, No. 3, 2007, pp. 938-944. doi:10.1016/j.na.2006.06.051

[4] N. Van Luong and N. X. Thuan, "Coupled Fixed Points in Partial Ordered Metric Spaces and Application," Nonlinear Analysis, Vol. 74, No. 3, 2011, pp. 983-992. doi:10.1016/j.na.2010.09.055

[5] N. Chen, and J. Q. Chen, "New Fixed Point Theorems for 1-Set-Contractive Operators in Banach Spaces," Nonlinear Analysis, Vol. 6, No. 3, 2011, pp. 147-162.

[6] G. Z. Li, "The Fixed Point Index and the Fixed Point Theorems of 1-Set-Contrac-Tive Mappings," Proceedings of the American Mathematical Society, Vol. 104, No. 4, 1988, pp. 1163-1170. doi:10.1090/S0002-9939-1988-0969052-9
[7] C. X. Zhu and Z. B. Xu, "Inequality and Solution of an Operator Equation," Applied Mathematics Letters, Vol. 21, No. 6, 2008, pp. 607-611. doi:10.1016/j.aml.2007.07.013

[8] R. Saadati, M. Dehghan, S. M. Vaezpour and M. Saravi, "The Convergence of He's Variational Iteration for Solving Integral Equations," Computers \& Mathematics with Applications, Vol. 58, No. 11-12, 2009, pp. 2167-2171. doi:10.1016/j.camwa.2009.03.008

[9] Y. F. Xu, "The Variational Iteration Method for Autonomous Ordinary Differential Equations with Fractional Order," Journal of Hubei University Nationalities (Nature Science Edition), Vol. 29, No. 3, 2011, pp. 245-249.

[10] G. B. Asghar and S. N. Jafar, "An Effective Modification of He's Variational Iteration Method," Nonlinear Analysis: Real World Application, Vol. 10, No. 5, 2009, pp. 2828-2833. doi:10.1016/j.nonrwa.2008.08.008

[11] J. H. He, "Variational Iteration Approach to Schrodinger Equation," Acta Mathematica Scienca, Vol. 21A, 2001, pp. 577-583.

[12] S .Q. Wang and J. H. He, "Variational Iterative Method for Solving Integro-Differential Equations," Physics Letters A, Vol. 367, No. 3, 2007, pp. 188-191. doi:10.1016/j.physleta.2007.02.049

[13] Z. Z. Zhang and S. R. Lu, "Numerical Solution of Schrodinger Equation," Journal of Shanxi Daton Universeity, Vol. 26, No. 2, 2010, pp. 22-24.

[14] Y. F. Wang and L. B. Tang, "Direct Solution of One-Dimensional Schrodinger Equation through Finite Difference and MATLAB Matrix Computation," INFRARED (MONTHLY), Vol. 31, No. 3, 2010, pp. 42-46. 